\title{
Scalp psoriasis and biofilms: electron microscopy
}

\begin{abstract}
Psoriasis is an immune-mediated inflammatory skin disease. When examining punch biopsies of a patient's scalp with psoriasis, we for the first time used scanning electron microscopy (SEM) in combination with the express method of transmission electron microscopy (TEM) - negative contrasting with phosphoric tungsten acid (FVA). The study compared biopsies from scalp affected and unaffected by psoriasis. It was shown that psoriatic areas of the scalp are covered with biofilms consisting of clusters of coccoid bacteria and yeast cells of fungi, connected by thin chaotically located strands. The number of hairs on the affected skin area with psoriasis was 2 times less than on the unaffected one. and the hair cuticle at the lesions was dystrophic.

The use of the TEM method revealed only yeast cells in the scalp scales, which, in terms of size, shape (the presence of a wide scar) and monopolar budding, allowed them to be attributed to the genus Malassezia. Fungi of the genus Malassezia were less common in the scales of the affected skin area, as compared to the unaffected one. This discrepancy in the results can be explained by the fact that the formed bacterial-mycotic biofilms, due to the extracellular matrix, affect the vital activity of fungi and prevent them from freely settling between the scales. Most likely, it is the combination of bacteria and fungi in the form of bacterial-mycotic films on the skin that initiates the appearance of psoriatic eruptions, and in further studies it is advisable to consider bacteria and fungi in combination. It is necessary to find out what factors contribute to the formation of such associations during the formation of biofilms, which will allow finding new methods of therapy.
\end{abstract}

Volume 8 Issue 5 - 2020

\author{
Amalia A Stepanova,Vera G Kornisheva, \\ Konstantin I Raznatovskiy, Olga A Smolina \\ North-Western State Medical University named after I.I. \\ Mechnikov, St. Petersburg, Russia
}

Correspondence: Vera G Kornisheva, North-Western State Russia, Email v.g.korniseva@gmail.com

Received: October 12, 2020 | Published: October 30, 2020

Keywords: biofilms, Malassezia, psoriasis, scalp, scanning and transmission electron microscopy

\section{Introduction}

Up to $3 \%$ of the world's population suffers from psoriasis, i.e. about 80 million people. ${ }^{1}$ In about $80 \%$ of patients with psoriasis with lesions of smooth skin, scalp lesions are also observed, however, in $25 \%$ of cases, psoriasis of the scalp occurs in isolation. ${ }^{1,2}$ The lesion of the scalp presents particular difficulties for the choice of topical therapy, and the presence of rashes on open areas of the skin and the aesthetic unattractiveness of lesions significantly reduce the quality of life of patients. ${ }^{2}$ The structural features of the skin of patients with psoriasis at the electronic microscopic level have not been sufficiently studied. ${ }^{3-5}$ They are mainly devoted to the study of the structure of the hair cuticle in patients with psoriasis in a scanning electron microscope (SEM). The data obtained are fragmentary and contradictory in terms of information content. It is believed that fungi of the genus Malassezia are the physiological microbiota of human skin, they can cause the overproduction of molecules in keratinocytes involved in cell migration and hyperproliferation, and thereby contribute to the exacerbation of psoriasis. ${ }^{6}$ It was of interest to conduct a detailed study of the affected and unaffected parts of the patient's scalp skin using modern SEM methods in order to identify the presence of structural changes in the scalp skin and its appendages in psoriasis.

\section{Materials and methods}

For the study, a punch biopsy of the scalp of a 43 -year-old patient with psoriasis was performed. The material was taken from the affected and unaffected parts of it.

\section{Method}

For scanning electron microscopy (SEM), the studied scalp biopsies were fixed in $3 \%$ glutaraldehyde (in cacodylate buffer, $\mathrm{pH}$ 7.2) for 3 hours, post-fixed overnight in $1 \%$ OsO4, dehydrated in a series of alcohols $(30 \% \rightarrow 50 \% \rightarrow 70 \%)$, and then dried at the critical point (HCP-2) for 15 minutes, sprayed with gold and studied in a JSM 35 SEM (Jeol, Tokyo, Japan).

For the first time, the express method of transmission electron microscopy (TEM) - negative contrasting with phosphoric-tungstic acid (FVA) - was tested to identify microorganisms directly in epidermal scales in order to describe the frequency of their occurrence and their morphology. To carry out transmission electron microscopy (TEM) - negative contrast with the staining of the FVA with a microbiological loop, a part of the epidermal scales collected from the affected and unaffected areas of the patient's scalp was transferred into sterile plastic tubes for Eppendorf microprobes $(1.5 \mathrm{ml})$ containing physiological solution $(0.1 \mathrm{ml})$ for 10 minutes. The resulting suspensions were applied with an automatic pipette on electron microscopic copper grids with a diameter of $3 \mathrm{~mm}$, covered with a transparent mold-varnished substrate-film. Then, a $1.5 \%$ solution of FVA ( $\mathrm{pH}$ 6.7) was applied to the grids with the material for 10 minutes, which stained the objects negatively in black and allowed TEM to clearly reveal the details of their ultrastructural organization. After staining with FVA, the grids were washed with distilled water, transferred to dry filter paper in a Petri dish, dried for 10 minutes, and examined in TEM Jem 100-SX (Jeol, Japan). 


\section{Ethical approval}

The study was approved by the ethical committee at North-Western State Medical University named after I.I. Mechnikov.

\section{Result}

\section{Affected skin}

The study of the structure of the surface of the epidermis between the hair follicles at a low magnification of a scanning microscope (Figure 1a) revealed the presence of tightly adjacent fine-warty formations of irregular shape, covered with biofilms of a specific structure. The latter are discrete, of different areas and shapes, of varying thickness, cover the surface of the epidermis in a mosaic manner (Figure 1b, arrows). At high SEM magnification, it can be seen (Figure 1c) that the biofilms consist of clusters of single or small groups of coccoid bacteria, as well as yeast cells of fungi, interconnected by thin $(0.08 \mu \mathrm{m})$ chaotically located strands (Figure $1 \mathrm{c}$, arrowheads). Bacteria dominated in biofilms. This type of films can be called bacterial-mycotic, with the predominance of the former. In this area ofthe scalp, the skin scales were mainly concentrated perifollicularly (Figure 1e).
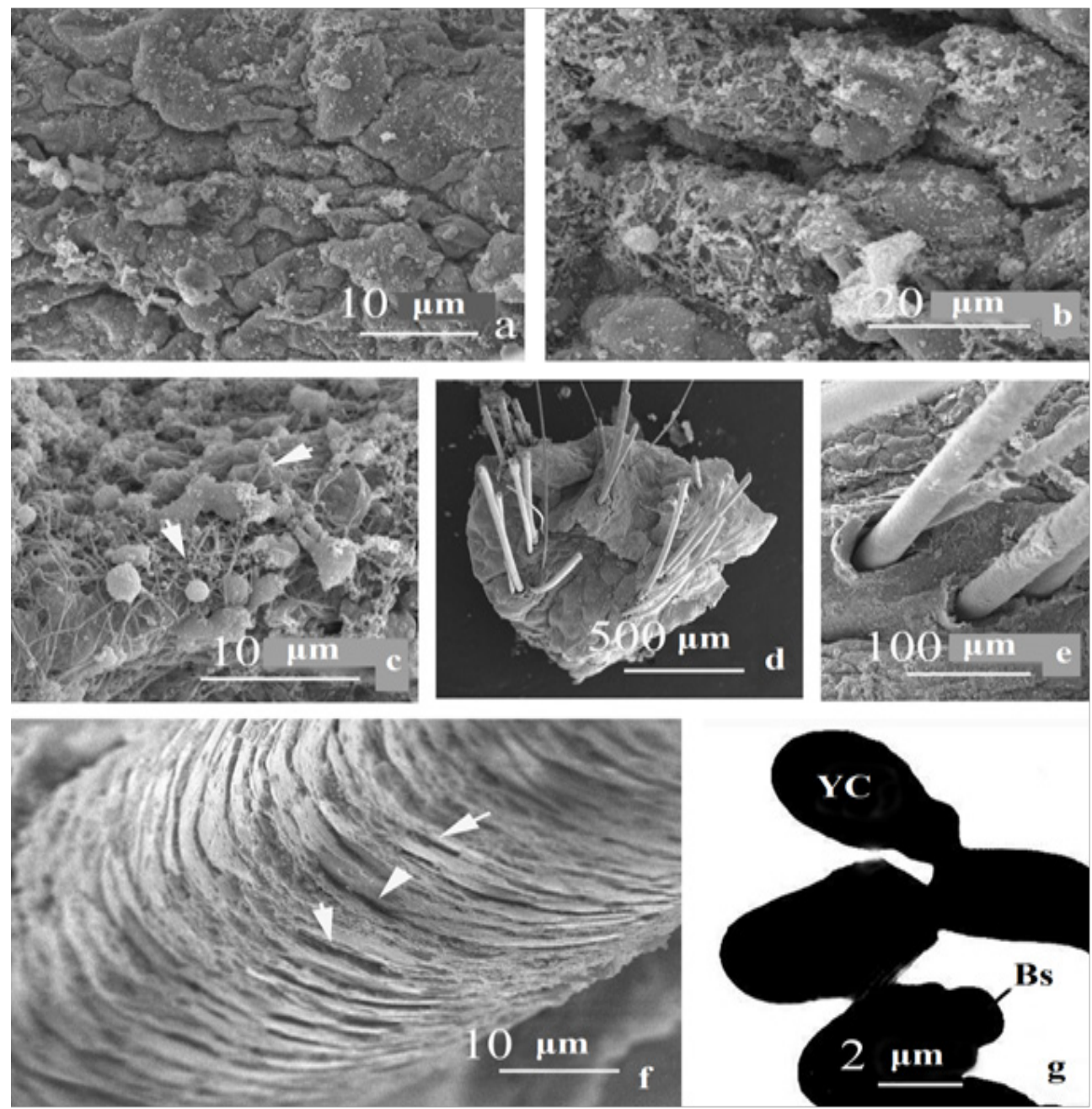

Figure I Features of the structure of the skin and its appendages of the affected area of the scalp of a patient with psoriasis. a-c - the surface of the epidermis between the hair follicles; $d$ - general view of the sample, e - hair funnel, $f$ - hair cuticle, $g$ - structure of Malassezia yeast cells in TEM after FVA staining. 


\section{Unaffected skin}

Fine-warty formations on the surface of the epidermis in the spaces between the hair follicles are absent (Figure 2a). The surface of the skin is uneven, repeats the contour of epidermal cells. Skin scales are presented in a greater number (Figure 2a, arrows), in comparison with the affected skin area. They are small, with uneven, often raised edges, which is characteristic of physiological desquamation.

The number of hairs in the skin of the biopsy is 20, they are located in grafts of 2-3 hairs each (Figure 2b). Hair diameter varied from 32.0 to $64.0 \mu \mathrm{m}$ (average $48.0 \mu \mathrm{m}$ ). Around the hair funnel of the analyzed sample, there are multilayer vertically arranged thin scales (Figure 2c, $2 \mathrm{~d}$, arrows), larger than near the hair funnels of the affected skin of the described case. Hair cuticle scales are well expressed, varying in thickness (1-3 microns), have a slightly uneven edge, tightly adjacent to the underlying scale (Figure 2e). In general, the surface structure of the hair has a "tiled appearance". No disturbances in the structure of the scalp hair cuticle of this variant were found. There were no biofilms on the scalp skin surface. Yeast cells in skin scales after FVA staining and study in TEM were observed extremely rarely, they are single or in small groups (Figure 2e). They are ellipsoidal (2.42.5x3.3-3.5 microns), rarely bud. Budding is monopolar, daughter cells are rounded.
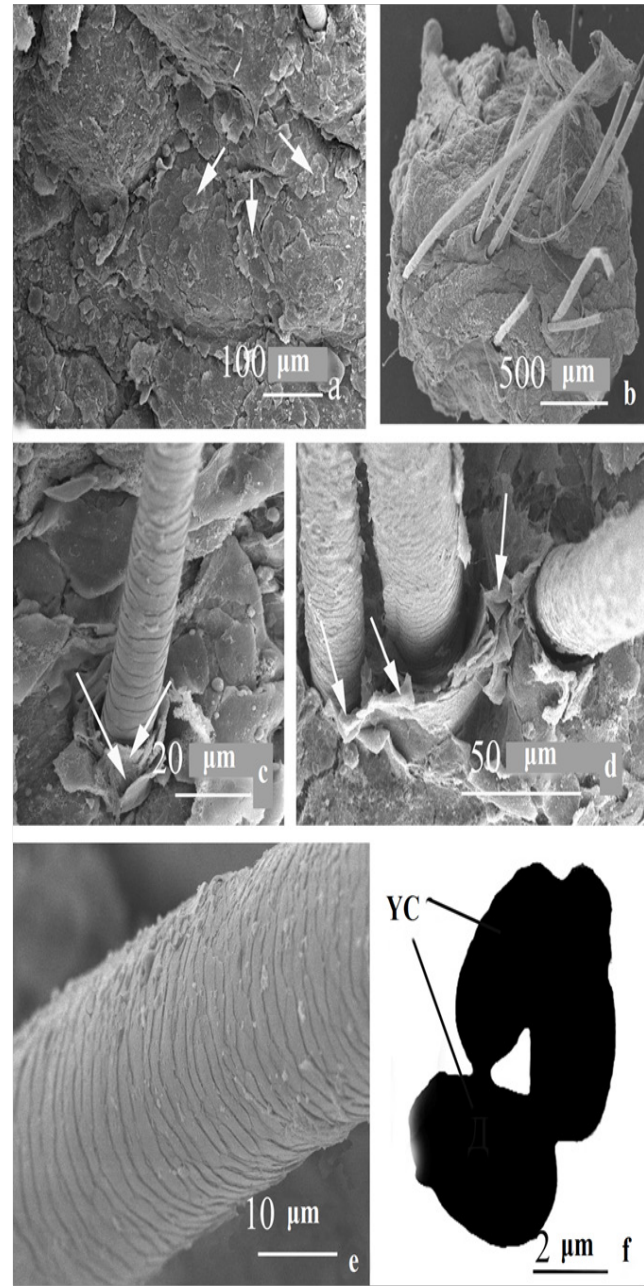

Figure $\mathbf{2}$ The structure of the skin and its appendages of the unaffected area of the scalp of a patient with psoriasis. a - the surface of the epidermis between the hair follicles; b - general view of the sample, c, $d$ - hair funnel, e - hair cuticle, $f$ - structure of Malassezia yeast cells in TEM after stainingwith FVA.

\section{Discussion}

The studies carried out using the SEM method showed that with psoriasis, changes occur both in the structure of the surface layer of the skin and its appendages. A specific feature of the structure of the epidermis of the skin in unaffected areas of the skin was the presence of numerous skin scales. At the same time, on the affected area of the skin, a small number of skin scales were found only in the area of the hair funnel.

On the affected area of the skin, the frequency of occurrence of hair was 2 times less than on the unaffected one. There were no significant differences in the mean hair diameter of the affected and unaffected areas of the scalp skin. The cuticle of the hair of the affected area, in comparison with the unaffected one, looked destroyed, dystrophic. Our studies are consistent with the literature data on the presence of serious dystrophic changes in the structure of the hair cuticle of the scalp affected by psoriasis. ${ }^{3-5}$ As the authors note, in the hair cuticle such dystrophic changes as its weak severity, "torn" edge, the presence of cracks or depressions of different depths are noted. The variety of dystrophic changes in the hair cuticle of the areas affected by psoriasis can be explained by their different ages, different duration and severity of the course of psoriasis in each specific case, as well as the patient's burden of other concomitant diseases, primarily the skin.

The skin surface of the affected area was covered with numerous bacterial mycotic biofilms. Similar bacterial mycotic films with thin strands in their composition were previously identified in the study of venous catheters. ${ }^{6}$ Often in biofilms, mucus is the main matrix, which is one of the important components in which microorganisms are immersed. In the studied biofilms, there was no mucus, but there were thin chaotically located cords of organic origin. Proteins secreted by bacteria act as the main structural component of the extracellular matrix, since they can self-organize into highly stable amyloid fibrils, which makes biofilm very difficult to degrade by physical and chemical means after formation. ${ }^{7}$ Previously, it was shown that biofilm consists of polysaccharide, protein and nucleic acid secreted by bacteria on the surface of biological material. Bacteria produce secondary metabolites, some of which act as signaling molecules, allowing bacteria to communicate and regulate many important physiological behaviors at the multicellular level, including biofilm formation. ${ }^{8}$ The formation of bacterial-mycotic films on the surface of psoriatic eruptions on the scalp in our patient creates optimal conditions for the survival of both bacteria and fungi and indicates their role in the formation of inflammation. This is confirmed by previous studies. On the one hand, it has been shown that Staphylococcus aureus biofilm can aggravate skin inflammation along the Th17 axis in psoriasis. At the molecular level, psoriasis is usually triggered by a Th17 response, which is the main therapeutic target. ${ }^{9}$ On the other hand, immunity to commensal skin fungi has been shown to promote psoriasis-like skin inflammation. Responses to commensal cutaneous fungi exacerbate tissue inflammation. ${ }^{10}$ The link between the skin fungus microbiome and psoriasis has yet to be established. When identifying fungal microbiota specific for psoriasis, it was revealed that Malassezia $s p p$. is the most common fungi. ${ }^{11}$ Most likely, it is the combination of bacteria and fungi in the form of bacterial-mycotic films on the skin that initiates the appearance of psoriatic eruptions, and in further studies it is advisable to consider these infections in combination. It is necessary to find out what factors contribute to the formation of such associations during the formation of biofilms, which will allow finding new methods of therapy.

In this study, for the first time, an attempt was made to use the TEM method of negative contrast with the help of FVA for the rapid 
detection and study of the microbiota of skin scales. The application of this method made it possible to reveal in the scalp scales only yeast cells, which in size, as well as the presence of a wide scar and monopolar budding, would allow them to be attributed to the genus Malassezia. ${ }^{12,13}$ Fungi of the genus Malassezia were less common in the scales of the affected skin area, as compared to the unaffected one. This is consistent with our mycological studies of skin scales in patients with scalp psoriasis. At the same time, the SEM method revealed fungi on the surface of psoriasis foci. This discrepancy in the results can be explained by the fact that the formed bacterial-mycotic films, due to the extracellular matrix, affect the vital activity of fungi and prevent them from freely settling between the scales. At the same time, the morphology of yeast cells in the scales of both affected and unaffected skin areas was similar.

The studies carried out demonstrate the promising use of the negative contrasting FVA method for diagnostic purposes, since it allows you to quickly and reliably identify the presence of fungi and bacteria in TEM directly as a biomaterial, as well as clarify some extremely important details of the ultrastructure that cannot be detected by classical cultural research methods.

\section{Conclusion}

To study bacterial-mycotic films on the skin, it is advisable to combine scanning electron microscopy of skin biopsies with the study of skin scales previously taken from these foci in TEM using the FVK staining method in order to detect microorganisms in them. The studies carried out demonstrate the promisingness of using the negative contrasting FVA method for diagnostic purposes, since it allows you to quickly and reliably identify the presence of fungi and bacteria in TEM directly as a biomaterial, as well as clarify some extremely important details of the ultrastructure that cannot be detected by classical cultural research methods.

\section{Acknowledgments}

None.

\section{Conflicts of interest}

The authors declare no conflicts of interest.

\section{References}

1. Bovenschen HJ, Van de Kerkhof PC. Treatment of scalp psoriasis with clobetasol-17 propionate $0.05 \%$ shampoo, a study on daily clinical practice. J Eur Acad Dermatol Venereol. 2010;24(4):439-444.

2. Ortonne J, Chimenti S, Luger T, et al. Scalp psoriasis: European consensus on grading and treatment algorithm. J Eur Acad Dermatol Venereol. 2009;23(12):1435-1444.

3. Plozzer C, Coletti C, Kokelj F, e al. Scanning electron microscopy study of hair shaft disorders in psoriasis. Acta Derm Venereol Suppl (Stockh). 2000;211:9-11.

4. Kumar B, Soni A, Saraswat A, et al. Hair in psoriasis: a prospective, blinded scanning electron microscopic study. Clin Exp Dermatol. 2008;33(4):491-494.

5. Rateb AA, Abdel-Aal WE, Shaffie NM, et al. Scaly scalp in different dermatological diseases: a scanning and transmission electron мicroscopical study. Nature and Sciences. 2010;8(12):61-69.

6. Stepanova AA, Vasilyeva NV, Pinegina ON. Scanning electron microscopy of biofilm of urethral and venous catheters. Problems of Medical Mycology. 2014;16(4):32-37.

7. Andreasen M, Meisl G, Taylor JD, et al. Physical Determinants of Amyloid Assembly in Biofilm Formation. mBio. 2019;10(1):e02279-e02318.

8. Chuntian Tu, Yang Wang, Li YI, et al. Roles of signaling molecules in biofilm formation. Sheng Wu Gong Cheng Xue Bao. 2019;35(4):558-566.

9. Hsin-Wen Chang, Di Yan, Rasnik Singh et al. Alteration of the cutaneous microbiome in psoriasis and potential role in Th17 polarization. Microbiome. 2018;6(1):154.

10. Hurabielle C, Link VM, Bouladoux N, et al. Immunity to commensal skin fungi promotes psoriasiform skin inflammation. Proc Natl AcadSci U S A. 2020;117(28):16465-16474.

11. Takemoto A, Cho O, Morohoshi Y. Molecular characterization of the skin fungal microbiome in patients with psoriasis. $J$ Dermatol. 2015;42(2):166-170.

12. de Hoog GS, Guarro J, Gené J, et al. Atlas of clinical fungi (a recent electronic version 3.1, 2019).

13. Boekhout T, Guého-Kellermann E, Mayser P, et al. Malassezia and the skin. Berlin: Springer; 2010. 319 p. 